\title{
M32: Is there an Ancient and Metal-poor Stellar Population?
}

\author{
Giuliana Fiorentino ${ }^{1}$, Antonela Monachesi ${ }^{1}$, Scott C. Trager ${ }^{1}$, \\ Tod R. Lauer ${ }^{2}$, Abhijit Saha ${ }^{2}$, Kenneth J. Mighell ${ }^{2}$, \\ Wendy Freedman ${ }^{3}$, Alan Dressler ${ }^{3}$, Carl Grillmair $^{4} \&$ Eline Tolstoy $^{1}$ \\ ${ }^{1}$ Kapteyn Astronomical Institute, \\ Postbus 800, 9700 AV Groningen, The Netherlands \\ email: fiorentino@astro.rug.nl \\ ${ }^{2}$ National Optical Astronomy Observatory, ${ }^{3}$ The Observatories of the Carnegie Institution of \\ Washington, ${ }^{4}$ Spitzer Science Center.
}

\begin{abstract}
We observed two fields near M32 with the ACS/HRC (Program GO-10572, PI: T. Lauer) on board the Hubble Space Telescope, located at distances of about 1.8' and 5.4' (hereafter F1 and F2, respectively) from the center of M32. To obtain a very detailed and deep color-magnitude diagram (CMD) and to look for short period variability, we obtained time-series imaging of each field in 32-orbit-long exposures using the F435W (B) and F555W (V) filters, spanning a temporal range of 2 days per filter. We focus on our detection of variability on RR Lyrae variable stars, which represents the only way to obtain information about the presence of a very old population (larger than $10 \mathrm{Gyr}$ ) in M32 from optical data. Here we present results obtained from the detection of 31 RR Lyrae in these fields: 17 in F1 and 14 in F2. We claim we detected $7_{-3}^{+4}$ RR Lyrae variables belonging to M32 in F1 thus indicating the presence of a metal-poor ancient population in M32.
\end{abstract}

Keywords. galaxies: elliptical: stars: variables: RR Lyrae

\section{Observation and data reduction}

The exposure time of each image is nearly 22 minutes. For each field and filter the time window is spread over 2-3 days, appropriate for searching for $\mathrm{RR}$ Lyrae stars $(\mathrm{P} \sim 0.2-1$ d). The super-resolved images obtained by combining all 32 images, for each filter and field, returned us the deepest CMD of M32 obtained so far (for figures and details see Monachesi's talk, this conference). Then to detect the variability we analyzed each single epoch images (32 per field and per filter) by using DOLPHOT package Dolphin(2000) optimized "ad hoc" for ACS images. The photometry then has been calibrated by following the suggestions by Sirianni et al.(2005). The identification and analysis were made by using a code written by one of us (AS) in the Interactive Data Language (IDL), Saha \& Hoessel(1990) and GRATIS (GRaphical Analyzer of TIme Series, a private software developed by P. Montegriffo at the Bologna Observatory; see Clementini et al.(2000). This procedure has allowed us to derive well sampled and consistent light curves (see an example in Fig. 1, left panel) for 17 RR Lyrae in F1 and 14 in F2. The RR Lyrae light curves as well as all their relevant pulsation properties (as period, mangnitude, colors, reddening, distance modulus, etc), are available in Fiorentino et al. 2009, (arXiv:0909.0202).

Here we just emphasize that the pulsation properties of RR Lyrae belonging to F1 and F2 do not show any statistically significant difference in term of the Poisson errors, due to the small samples (FoV of HRC is $\left.0.25^{\prime \prime 2}\right):\langle V\rangle=25.34 \pm 0.15 \mathrm{mag}, N_{c} / N_{a b}=0.30_{-0.30}^{+0.37}$ for $\mathrm{F} 1$ and $\langle V\rangle=25.30 \pm 0.12 \mathrm{mag}, N_{c} / N_{a b}=0.75_{-0.42}^{+1.25}$ for F2. For both fields FO 

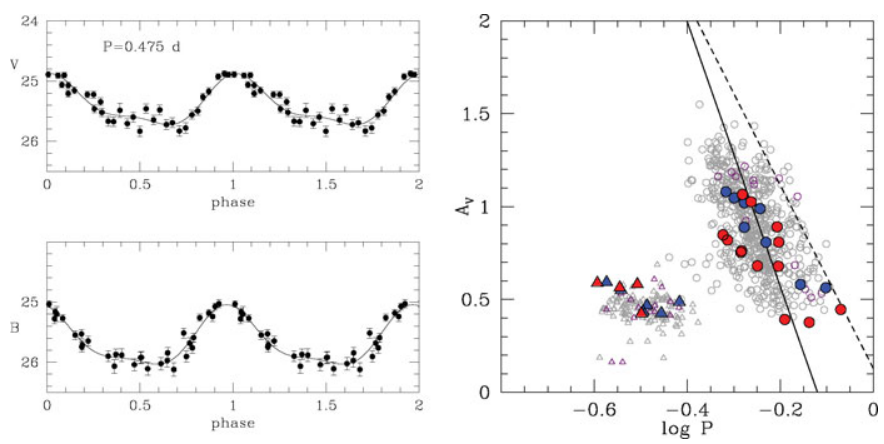

Figure 1. Left panel: an example of the B and V light curves. Right Panel: The Bailey Diagram. RR Lyrae stars in F1 (dark grey), in F2 (black), in Sarajedini et al.(2009) and in Brown et al.(2004) (light grey). FU and FO pulsators are circles and triangles. Solid and dashed lines are Oosterhoff types I and II from Clement(2000). Nearly all FU pulsators follow the OoI relation.

pulsators have mean periods of $\sim 0.3 \mathrm{~d}$ and sinusoidal light curves, whereas the FU pulsators have longer periods $\left(\left\langle P_{a b}\right\rangle=0.59 \pm 0.11 \mathrm{~d}\right)$ and more complicated light curves (with up to 4 harmonics). Amplitudes and periods have been shown in Fig. 1 (right panel), where we also compare our RR Lyrare to the ones found in the Halo/Disk of M31 from Brown et al.(2004) and Sarajedini et al.(2009) (light grey, hereinafter fields F3-F4).

\section{Implications}

We then asked whether we detected RR Lyrae stars belonging on M32 or just to the Halo/Disk of M31. Unfortunately the low statistic of our samples and the high background contamination from M31 play a key role into the answering to this question.

We examined the implications of our detections of RR Lyrae variables in F1 and F2, by assuming as M31 background first only F2 field and second an average made on F2-F3-F4 fields. This analysis allows us to claim we detected $7_{-3}^{+4} \mathrm{RR}$ Lyrae variables belonging to M32 in field F1 under the second assumption. Therefore we conclude that, indeed, M32 has an ancient, metal-poor population as represented by the detection of RR Lyrae stars (all the details can be found in Fiorentino et al. 2009, submitted).

\section{References}

Brown, T. M., Ferguson, H. C., Smith, E., Kimble, R. A., Sweigart, A. V., Renzini, A., \& Rich, R. M. 2004, AJ, 127, 2738

Clement, C. M. 2000, in Astronomical Society of the Pacific Conference Series, Vol. 203, IAU Colloq. 176: The Impact of Large-Scale Surveys on Pulsating Star Research, ed. L. Szabados \& D. Kurtz, 266

Clementini, G., et al. 2000, AJ, 120, 2054

Dolphin, A. E. 2000, PASP, 112, 1383

Saha, A. \& Hoessel, J. G. 1990, AJ, 99, 97

Sarajedini, A., Mancone, C. L., Lauer, T. R., Dressler, A., Freedman, W., Trager, S. C., Grillmair, C., \& Mighell, K. J. 2009, AJ, 138, 184

Sirianni, M., et al. 2005, PASP, 117, 1049

Stetson, P. B. 1987, PASP, 99, 191

-. 1994, PASP, 106, 250 